\title{
Superparamagnetic Behavior in Fe Ultrathin Films on GaN(0001)
}

\author{
P. K. J. Wong ${ }^{1,3}$, W. Zhang ${ }^{1}$, X. G. Cui ${ }^{2}$, I. G. Will', Y. B. Xu ${ }^{1,}$, Z. K. Tao ${ }^{2}, \mathrm{X} \mathrm{Li}^{2}$, Z. L. Xie ${ }^{2}$ and R. Zhang ${ }^{2}$ \\ ${ }^{1}$ Spintronics and Nanodevice Laboratory, Department of Electronics, University of York, York, \\ YO10 5DD, United Kingdom \\ ${ }^{2}$ Jiangsu Provincial Key Laboratory of Advanced Photonic and Electronic Materials and \\ Department of Physics, Nanjing University, Nanjing 210093, People's Republic of China \\ ${ }^{3} \mathrm{MESA}^{+}$Institute for Nanotechnology, University of Twente, P.O. Box 217, 7500 AE Enschede, \\ The Netherlands \\ *Correspondence author: yx2@ohm.york.ac.uk
}

Here we report on the studies on the growth, structural and magnetic properties of ultrathin $\mathrm{Fe}$ grown on $\mathrm{GaN}(0001)$ by molecular beam epitaxy. The films and their surfaces were monitored by in-situ reflection high energy electron diffraction (RHEED) and a crystal thickness monitor. The magnetic properties of the samples were determined by a superconducting quantum interference device (SQUID) magnetometer. The $2 \mu \mathrm{m}$ thick GaN films used were prepared by metal-organic chemical vapor deposition (MOCVD) with a $20 \mathrm{~nm}$ low temperature (LT) buffer layer on $\mathrm{c}-\mathrm{Al}_{2} \mathrm{O}_{3}(0001)$. Prior loading into vacuum, the $\mathrm{GaN}$ surface was cleaned in a 1:1 $\mathrm{HCl}: \mathrm{H}_{2} \mathrm{O}$ solution then a 1:99 $\mathrm{HF}: \mathrm{H}_{2} \mathrm{O}$ solution for 2 min each with DI water rinses between and after etching. This ex-situ chemical processes combined with a vacuum anneal are useful to remove oxygen and carbon contaminants on the GaN surface [1]. After annealing at $600{ }^{\circ} \mathrm{C}$ for 1 $\mathrm{h}$ followed by cooling to room temperature $(\mathrm{RT})$, streaky and sharp bulk-terminated $(1 \times 1)$ RHEED patterns were obtained, indicating a smooth $\mathrm{GaN}$ surface. Fe films with various thicknesses ranging from 2.5 to $150 \mathrm{ML}$ were grown at RT at a chamber pressure of $1 \times 10^{-10} \mathrm{mbar}$ by $e$-beam evaporation at a rate of $2 \AA \mathrm{min}^{-1}$. Before samples retrieval for $e x$-situ characterization, the samples were capped with a $3.0 \mathrm{~nm}$ thick Au layer.

Evolution of the $\mathrm{Fe}(110)$ growth has been studied in-situ with the RHEED patterns as a function of Fe film thickness with the incident $e$-beam along the [11 20$]$ of the GaN(0001). The streaky $(1 \times 1)$ pattern of the clean $\mathrm{GaN}(0001)$ surface disappears upon a Fe coverage of $2.5 \mathrm{ML}$, indicating that the growth proceeds via a $3 \mathrm{D}$ mechanism as previously reported by He et al [2]. The faint main streaks of Fe exhibiting a bcc structure start to emerge at a deposition of 8 ML. Clear RHEED streaks of the Fe film become unambiguous at the deposition of $10 \mathrm{ML}$ from where onward, less intense reflexes are visible beside the main streaks and the Fe patterns appear spotty.

We further demonstrate that by reducing $t_{\mathrm{Fe}}$ down to a few ML, superparamagnetism (SPM) of the ultrathin Fe can be activated at the ambient temperature. Fig. 1(a) illustrates the hysteresis loop of an as-deposited $5 \mathrm{ML} \mathrm{Fe}(110)$ film on $\mathrm{GaN}(0001)$ taken at RT and reveals that the loop on one hand has an unsaturated magnetization and on the other hand possesses tiny but noticeable $M_{r}$ and $H_{c}$. These two characteristics as a whole imply a coexistence of SPM and weak FM in the ultrathin film. In order to gain further insight into this mixed magnetic state, temperature dependence of the magnetization $M(T)$ in the form of field cooling (FC) and zero field cooling (ZFC) curves of the 5 ML sample was measured by SQUID in a $T$ range between 5 and $300 \mathrm{~K}$ as illustrated in Fig. 1(b). The ZFC magnetization curve was performed by applying a small in-plane field of $300 \mathrm{Oe}$ to the sample at $5 \mathrm{~K}$ and then warming it with $M$ being measured 
as a function of $T$. Similarly, the FC curve was done by cooling the sample to $5 \mathrm{~K}$ from $300 \mathrm{~K}$ in the presence of the same field strength. Notable in Fig. 6(b) is irreversibility of the ZFC and FC curves below $120 \mathrm{~K}$, as indicated by the cusplike feature in the ZFC curve. This $T$ value at which the $M$ maximum occurs represents the blocking temperature $T_{B}$ of the $5 \mathrm{ML} \mathrm{Fe}$. More details will be given in the full manuscript.
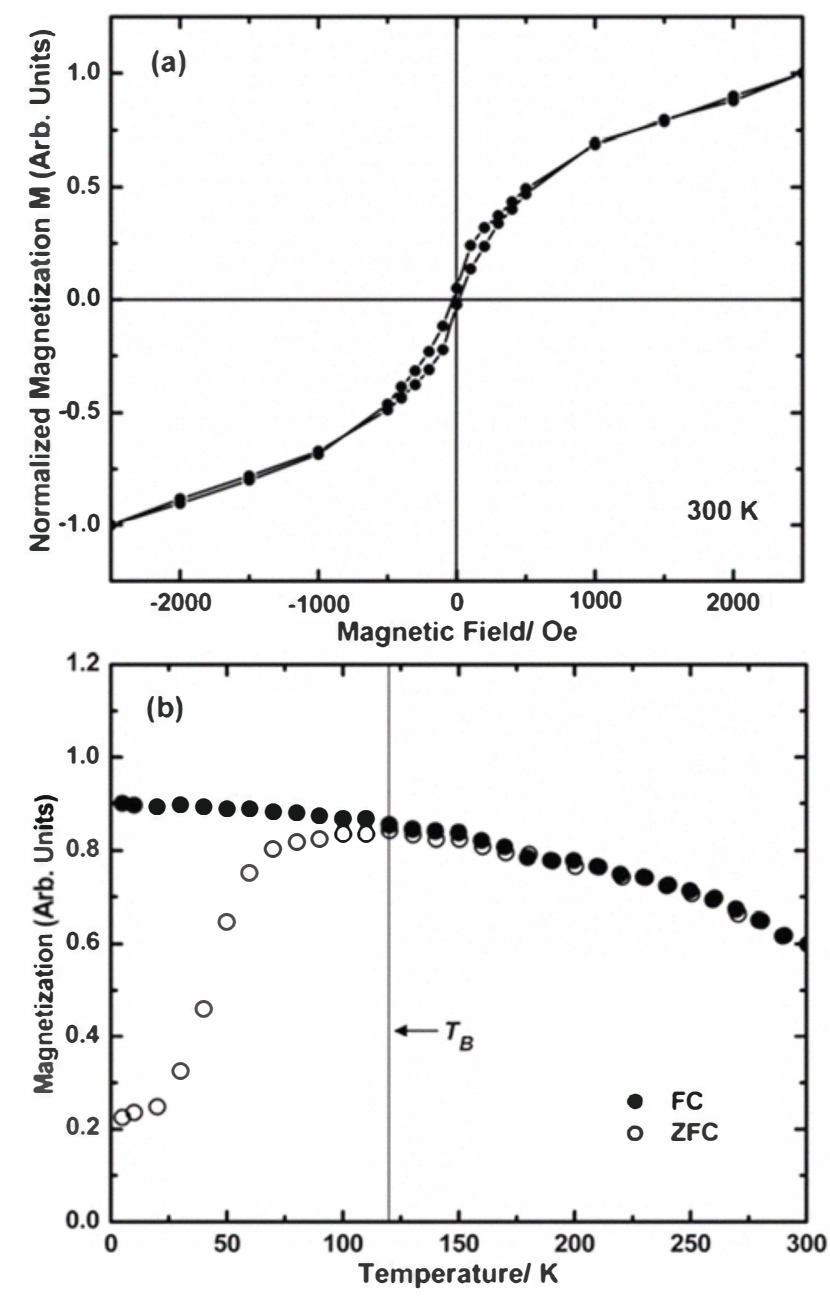

Fig.1 Magnetic hysteresis loop of a 5 ML thick Fe(110) film with an external field of 300 Oe applied along the [11 20 ] direction of the GaN(0001) at RT and (b) the corresponding ZFC and FC magnetization curves. The vertical line in (b) refers to the estimated blocking temperature $T_{B}$ of the Fe/GaN sample.

\section{References}

[1] L. L. Smith, S. W. King, R. J. Nemanich and R. F. Davis, J. Electron. Mater. 25, 805 (1996).

[2] K. He, L. Y. Ma, X. C. Ma, J. F. Jia and Q. K. Xie, Appl. Phys. Lett. 88, 232503 (2006). 